\title{
New Insight into the Hypercoagulability of Cushing's Syndrome
}

\author{
Viviana Daidone $^{a}$ Marco Boscaro ${ }^{c}$ Elena Pontara ${ }^{a}$ Maria Grazia Cattinia \\ Gianluca Occhi $^{\mathrm{b}}$ Carla Scaroni $^{\mathrm{b}}$ Franco Mantero $^{\mathrm{b}}$ Alessandra Casonato $^{\mathrm{a}}$ \\ aDepartment of Cardiologic, Thoracic and Vascular Sciences, Second Chair of Internal Medicine, \\ ${ }^{b}$ Department of Medical and Surgical Sciences, Endocrinology Unit, University of Padua Medical School, Padua, and \\ 'Division of Endocrinology, Department of Internal Medicine, Polytechnic University of Marche, Ancona, Italy
}

\section{Key Words \\ Cushing's syndrome $\cdot$ Von Willebrand factor • \\ Hypercoagulable state of Cushing's syndrome • \\ Von Willebrand factor promoter single nucleotide \\ polymorphisms • Von Willebrand factor promoter $(\mathrm{GT})_{\mathrm{n}}$}

\begin{abstract}
Background: Hypercoagulability and a tendency for thromboembolic complications are reported in Cushing's syndrome (CS). The hypercoagulability is due mainly to the cortisol-induced increase in von Willebrand factor (VWF) and factor VIII. This is not a constant feature of CS, however; it depends on particular single nucleotide polymorphism (SNP) haplotypes in the VWF gene promoter: haplotype 1 $(-3268 \mathrm{G} /-2709 \mathrm{C} /-2661 \mathrm{~A} /-2527 \mathrm{G})$ confers a greater risk of VWF upregulation by cortisol than haplotype $2(-3268 \mathrm{C} /$ $-2709 T /-2661 G /-2527 A)$. In healthy individuals these SNPs are in linkage disequilibrium with the $-2144(\mathrm{GT})_{n}$ of the VWF promoter: haplotype 1 mainly segregates with short GT repeats (15-19, GTs), haplotype 2 with long repeats (GT $\geq 20$, $\mathrm{GT}_{\mathrm{L}}$ ). Methods: We analyzed the $(\mathrm{GT})_{\mathrm{n}}$ locus, the SNP haplotypes and their association with VWF levels in 80 CS patients in order to precisely define the cortisol-sensitive VWF promoter pattern. CS patients were divided into groups $A$ (increased VWF) and B (normal VWF). Results: Haplotype 1 and $(\mathrm{GT})_{S}$ were more frequent in group $A$ patients, and conferred
\end{abstract}

a 9- and 7.5-fold risk of developing high VWF levels, respectively. Haplotype 2 and $(G T)_{\llcorner}$were more represented in group B. There was also an unexpected higher prevalence of recombinant SNP haplotypes in CS patients $(6.2 \%)$ than in normals $(0.9 \%), p=0.002$. Conclusions: Our results indicate that the cortisol-induced increase in VWF may be predicted by VWF promoter polymorphisms, haplotype 1 and $(\mathrm{GT})_{S}$ being the sensitive pattern. These represent new markers for defining the prothrombotic risk of CS. The clinical significance, if any, of the increased recombination rate in SNP haplotypes in the VWF promoter warrants further study.

Copyright $\odot 2011$ S. Karger AG, Basel

\section{Introduction}

Cushing's syndrome (CS) is characterized by an increase in morbidity and mortality, due mainly to cardiovascular complications associated with hypertension, obesity and glucose intolerance, but also to an increased incidence of thromboembolic events (both postoperative and spontaneous) related to the associated hypercoagulability [1-3]. CS hypercoagulability is due to a rise in clotting factors, especially von Willebrand factor (VWF) and factor VIII (FVIII) and, to a lesser extent, to a defective fibrinolysis, always correlating with the excessive cortisol secretion [4-6].

\section{KARGER}

Fax +4161306 1234

E-Mail karger@karger.ch

www.karger.com
(C) 2011 S. Karger AG, Basel

0028-3835/11/0932-0121\$38.00/0

Accessible online at:

www.karger.com/nen
Alessandra Casonato

Department of Cardiologic, Thoracic and Vascular Sciences

University of Padua Medical School

via Ospedale Civile 105, IT-51875 Padova (Italy)

Tel. +39 049821 3012, Fax +39 049657 391, E-Mail sandra.casonato@ unipd.it 
VWF is a multimeric glycoprotein mediating platelet adhesion at the site of vascular injury and stabilizing FVIII in the circulation [7]. The normal range of VWF levels is extremely wide, VWF concentrations being modulated by several genetic and environmental factors, and particularly the ABO blood group, individuals with group O having 25\% lower VWF levels than those with the other blood groups [8]. Although cortisol is known to upregulate VWF levels, CS patients are found to have normal VWF, despite their high urinary free cortisol (UFC) levels [9]. Indeed, the VWF response to glucocorticoid (GC) excess proved to be modulated by the single nucleotide polymorphisms (SNPs) -3268 G/C, -2709 $\mathrm{C} / \mathrm{T},-2661 \mathrm{~A} / \mathrm{G}$ and $-2527 \mathrm{G} / \mathrm{A}$ on the VWF gene promoter. Such SNPs are in linkage disequilibrium (LD), i.e. although many combinations could be expected on the basis of the product of single allele frequencies, only two patterns are actually seen in the population, i.e. haplotype 1 (GCAG) and haplotype 2 (CTGA) [10, 11]. In CS, the presence of haplotype 1 is associated with an increased risk of developing high VWF levels and a consequent hypercoagulable condition, while haplotype 2 correlates with normal VWF levels and thus protects against thrombotic complications [9]. The LD region in the VWF promoter also includes the variable-length $-2144(\mathrm{GT})_{\mathrm{n}}$ polymorphism, segregating with the SNP haplotypes in two main patterns, (GT) $)_{15-19}$ haplotype 1 and $(\mathrm{GT})_{20-24}$ haplotype 2 [12]. The $(\mathrm{GT})_{\mathrm{n}}$ locus has been found involved in the transcriptional modulation of VWF levels in response to shear-stress conditions, long $(\mathrm{GT})_{23}$ being associated with a stronger promoter activity than the shorter $(\mathrm{GT})_{17}$ [13]. In this work, the VWF gene promoter polymorphisms were analyzed in a group of CS patients to evaluate the contribution of both SNP haplotype and $(\mathrm{GT})_{\mathrm{n}}$ locus in cortisol-induced VWF upregulation.

\section{Materials and Methods}

\section{Subjects}

Patients and controls were studied after obtaining their written informed consent in accordance with the Helsinki Declaration and the study was approved by our institutional review board. 80 CS patients were studied (15 males and 65 females, mean age $43.7 \pm 13.5$ years), all followed up at the Endocrinology Unit of Padua Medical School. The patients were all in an active phase of the disorder and were not receiving any medication. 61 patients had CS of pituitary origin, and 19 had adrenal forms. CS was diagnosed on the basis of clinical features and hormonal assessments, as reported elsewhere $[9,14]$. A group of 160 normal individuals matched for gender and age was also studied, drawn from the Padua blood bank.
The CS patients were divided into groups A (high VWF levels) and B (normal VWF levels). Since the ABO blood group is the main genetic determinant of VWF levels, two different normal VWF ranges were used, i.e. 62-116 U/dl for O blood group subjects, and 67-171 U/dl for non-O cases, based on values previously calculated in 160 normal individuals [9].

\section{Hemostatic and Endocrine Analysis}

Plasma VWF antigen (VWF:Ag) levels were measured using a home-made enzyme-linked immunosorbent assay (ELISA), as reported previously [15]. UFC was evaluated by RIA, using a commercial kit (DIA-Sorin Diagnostics, Saluggia, Italy).

\section{Genetic Analysis}

Genomic DNA was extracted from peripheral blood leukocytes using the QIAamp ${ }^{\circledR}$ DNA Blood Mini Kit (Qiagen, Hilden, Germany). SNPs -3268G/C (rs7966230), -2709C/T (rs7964777), $-2661 \mathrm{~A} / \mathrm{G}$ (rs7954855), -2527G/A (rs7965413) and the -2144 $(\mathrm{GT})_{\mathrm{n}}$ locus of the VWF gene promoter were analyzed from genomic DNA, using an ABI3100 Genetic Analyzer (Applied Biosystems, Foster City, Calif., USA), as explained elsewhere [9, 12].

\section{Statistical Analysis}

The unpaired two-sample $t$ test and Fisher's exact test were used to compare patients in groups $\mathrm{A}$ and $\mathrm{B}$ with regard to age, sex, $\mathrm{ABO}$ distribution, glycemia levels and hypertension, nature of the disease, VWF and CLU levels. The $\chi^{2}$ test was used to compare allele and genotype distributions between groups A and B, and between patients and controls, for both $(\mathrm{GT})_{\mathrm{n}}$ locus and SNP haplotypes.

\section{Results}

There were 45 CS patients in group A (see Materials and Methods) (mean VWF $213.3 \pm 10.5 \mathrm{U} / \mathrm{dl}$ ), and 35 in group B (mean VWF $125.0 \pm 4.5 \mathrm{U} / \mathrm{dl}$ ). The two patient groups were comparable in terms of mean age, sex, nature of their disease and $\mathrm{ABO}$ blood group distribution, and their UFC levels were also similar. The two groups were similar in terms of glycemia levels and the presence of hypertension, which are also known to contribute to VWF upregulation (table 1).

\section{Contribution of the VWF Gene Promoter to \\ Cortisol-Induced Upregulation of VWF}

GT Repeat. $(\mathrm{GT})_{\mathrm{n}}$ locus analysis showed that $(\mathrm{GT})_{19}$ and $(\mathrm{GT})_{21}$ were the two most frequent alleles, in both normals and CS patients, but there was a different allele distribution in groups A and B: alleles with 15-19 repeats - $(\mathrm{GT})_{S}$ - were more frequent in group A, while variants with 20 repeats or more - $(\mathrm{GT})_{\mathrm{L}}$ - were more common in group B (fig. 1a). As for genotype, $(\mathrm{GT})_{\mathrm{S}} /$ $(\mathrm{GT})_{S}$ was common in group A (19.5\%), but rare in group B $(6.3 \%)$, while $(\mathrm{GT})_{\mathrm{L}} /(\mathrm{GT})_{\mathrm{L}}$ was more represented in 

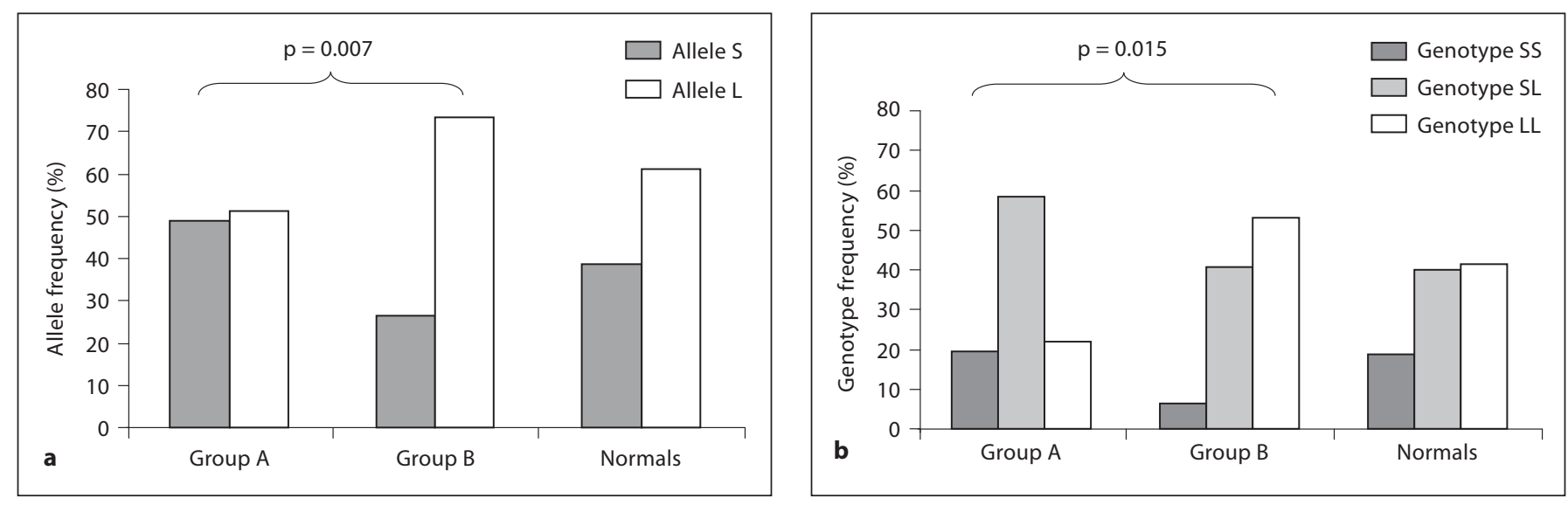

Fig. 1. Allele (a) and genotype (b) frequencies for the $(G T)_{n}$ locus in patients and normals. 'S' stands for $(G T)_{S}$ (15-19 GT repeats), 'L' stands for (GT) $)_{\mathrm{L}}$ (20-24 GT repeats). Both allele and genotype distribution differed significantly between the two groups of patients $(\mathrm{p}=0.007$ and $\mathrm{p}=0.015$, respectively).

group B (53.1\%) than in group A (22.0\%) (fig. 1b). The difference in haplotype and genotype distributions between groups A and B was statistically significant $(\mathrm{p}=0.007$ and $\mathrm{p}=0.015$, respectively), while no relevant difference was found between each patient group and normals. The calculation of the odds ratio (OR) revealed that the risk of developing high VWF levels in response to GC excess in CS was 2.6 times greater in patients carrying $(\mathrm{GT})_{\mathrm{S}}$ than in those carrying $(\mathrm{GT})_{\mathrm{L}}$ alleles $(\mathrm{OR}=2.6,95 \% \mathrm{CI} 1.3-5.3)$ and 7.5 times higher in homozygous $(\mathrm{GT})_{S} /(\mathrm{GT})_{S}$ than $(\mathrm{GT})_{\mathrm{L}} /(\mathrm{GT})_{\mathrm{L}}$ subjects $(\mathrm{OR}=7.5,95 \%$ CI 1.3-43.4).

SNP Haplotypes. Among the CS patients, there were 58 haplotype-1, 92 haplotype-2 and 10 recombinant patterns, represented by SNP combinations other than the wild types 1 and 2. Haplotype 1 (GCAG) was more frequent in group $\mathrm{A}$ than in group $\mathrm{B}$, whereas haplotype 2 (CTGA) was more represented in group B (fig. 2a). $\chi^{2}$ analysis revealed a statistically significant difference in both haplotype and genotype distribution between groups $\mathrm{A}$ and $\mathrm{B}(\mathrm{p}=0.002$ and $\mathrm{p}=0.027$, respectively). Haplotype 1 was associated with a 3.5-fold risk of developing high VWF levels by comparison with haplotype 2 $(\mathrm{OR}=3.5,95 \% \mathrm{CI} 1.7-7.5)$, and this risk became 9 times higher in CS patients homozygous for genotype $1 / 1$ than in the case of genotype $2 / 2(\mathrm{OR}=9.4,95 \%$ CI $1.7-52.7)$ (fig. 2b).

The frequency of recombinant SNP haplotypes was unexpectedly higher in CS patients (6.2\%) than in the healthy population $(0.9 \%)(\mathrm{p}=0.002)$. Recombinant patterns, distributed in both patient groups (A and $\mathrm{B})$ were: 4 CCAG, 3 GTGA, 1 GTAG, 1 CTGG, 1 GCGA (the nu-
Table 1. Main features observed in the CS patients studied

\begin{tabular}{lccl}
\hline & Group A & Group B & p values \\
\hline Patients, n & 45 & 35 & \\
VWF:Ag, U/dl & $213.3 \pm 10.5$ & $125.0 \pm 4.5$ & $<0.0001^{* *}$ \\
UFC, nmol/24 h & $1,069.0 \pm 126.3$ & $1,118.2 \pm 150.3$ & $0.80^{* *}$ \\
Pituitary/adrenal CS & $35 / 10$ & $27 / 8$ & $1.00^{*}$ \\
Age (mean \pm SD) & $46.2 \pm 15.3$ & $40.7 \pm 10.5$ & $0.0985^{* *}$ \\
Male/female & $10 / 35$ & $6 / 29$ & $0.779^{*}$ \\
O/non-O group & $21 / 24$ & $12 / 23$ & $0.360^{*}$ \\
Glycemia, mmol/1 & $5.9 \pm 0.4$ & $5.3 \pm 0.4$ & $0.2516^{* *}$ \\
Hypertension, yes/no & $38 / 7$ & $24 / 11$ & $0.111^{*}$ \\
\hline
\end{tabular}

$\mathrm{UFC}=$ Urinary free cortisol (values are given as mean \pm SEM); VWF $=$ von Willebrand factor (values are given as mean \pm SEM). ${ }^{*}$ Fisher's exact test. ${ }^{*}$ Unpaired t test.

cleotide not conforming with LD is underlined). Three CCAG haplotypes were found in the 160 healthy subjects studied.

\section{Discussion}

CS is characterized by a complex coagulative picture associated with a hypercoagulability that strongly contributes to these patients' high cardiovascular risk [3].

This hypercoagulability is characterized mainly by increased VWF levels that correlate well with these patients' increased cortisol levels [4] and the presence of un- 

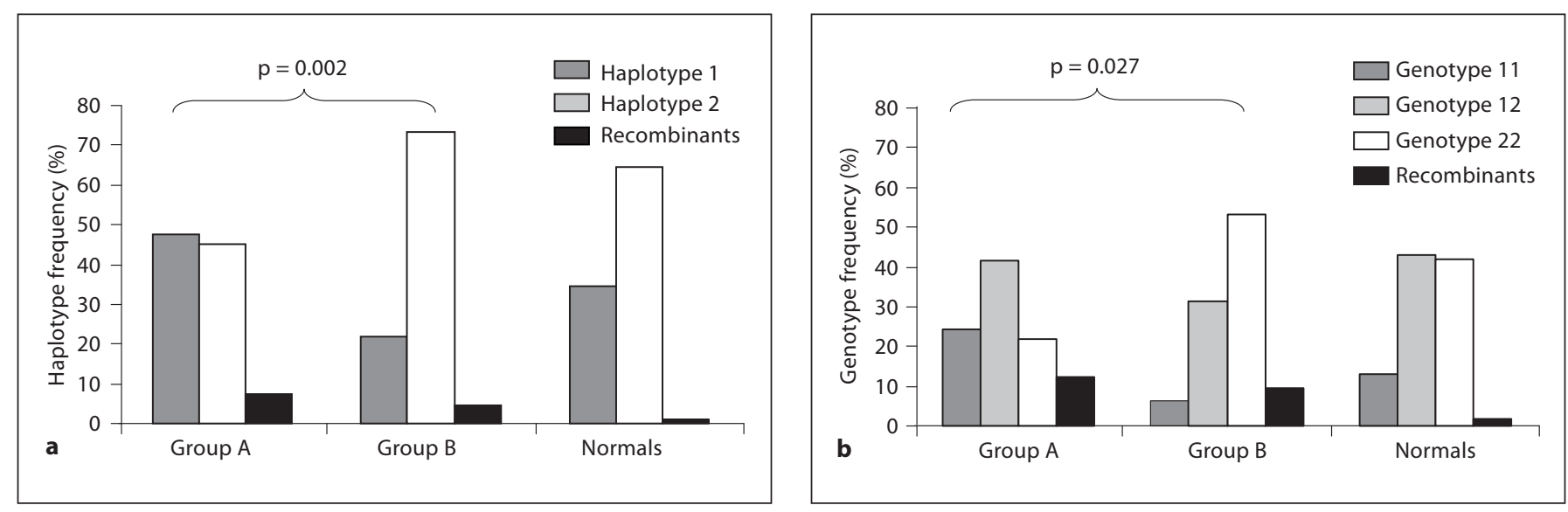

Fig. 2. Haplotype (a) and genotype (b) frequencies for SNPs -3268/-2709/-2661/-2527 in patients and normals. Haplotype 1 is GCAG, haplotype 2 is CTGA, while recombinants indicate any combination of polymorphisms other than haplotypes 1 and 2. A statistically significant difference was found between group A and group B patients for both haplotype and genotype distributions $(p=0.002$ and $p=0.027$, respectively). Note the higher frequency of recombinant patterns in CS patients than in the healthy population $(\mathrm{p}=0.002)$.

usually large VWF multimers, which are known to have the greatest hemostatic capacity [16]. VWF abnormalities are not a constant feature of CS, however. How VWF responds to cortisol excess has recently been shown to depend on the SNPs $-3268 \mathrm{G} / \mathrm{C},-2709 \mathrm{C} / \mathrm{T},-2661 \mathrm{~A} / \mathrm{G}$ and $-2527 \mathrm{G} / \mathrm{A}$ in the VWF gene promoter [9].

The results of this study confirm that the presence of haplotype 1 (GCAG) confers a 3.5 times higher risk of VWF increasing in CS than haplotype 2 (CTGA), and this risk is 9 times higher in patients homozygous for genotype $1 / 1$ than in the case of genotype $2 / 2$.

CS patients were found to have a higher frequency of recombinant SNP haplotypes than the general population. $\underline{C} C A G$ and GTGA were the most frequent recombinants, with the nucleotide at -3268 differing from the common patterns (GCAG and CTGA, respectively). This may be interpreted as the consequence of the greater physical distance of the -3268 SNP from the others (-2709, -2661 and -2527$)$, which makes a recombination separating the -3268 from the other three SNPs more likely. Be that as it may, we currently have no data to clarify the meaning of the higher recombination rate in the VWF promoter of CS patients, or whether this phenomenon is limited to the VWF gene or related to a more general impairment.

As for the $(\mathrm{GT})_{\mathrm{n}}$ locus, a different allele distribution was observed between the two patient groups: $(G T)_{S}$ variants were preferentially associated with group $A$ (patients with high VWF levels), and $(\mathrm{GT})_{\mathrm{L}}$ with group B (normal
VWF). No significant difference emerged in the allele and genotype distribution between the patient groups and normals, instead CS patients carrying the $(\mathrm{GT})_{S}$ allele showed a risk of developing high VWF levels that was 2.6 times higher than for those carrying the $(G T)_{L}$ allele, and 7.5 times higher for cases of $(\mathrm{GT})_{S} /(\mathrm{GT})_{S}$ than for $(\mathrm{GT})_{\mathrm{L}} /(\mathrm{GT})_{\mathrm{L}}$. So, like the SNP haplotypes, the $(\mathrm{GT})_{\mathrm{n}}$ locus also predicts each CS patient's risk of VWF increasing in response to $\mathrm{GC}$ excess.

The role of $(\mathrm{GT})_{\mathrm{S}}$ alleles as risk factor for VWF increasing in CS seems to contrast with the observation that the presence of long $(\mathrm{GT})_{\mathrm{n}}$ confers a greater VWF promoter activity under shear stress conditions [13]. This apparent discrepancy may be explained by the fact that different conditions were considered (shear stress vs. cortisol excess); short GT repeats may therefore be involved in different regulatory pathways, acting as an up- or downregulator of VWF gene transcription depending on whether the stimulus is cortisol or high shear stress, respectively. The validity of our observations seems to be confirmed by the finding that the GT repeat on the VWF promoter separates two hypothetical half-glucocorticoid response element (GRE) hexamers, making the whole GRE sequence more or less close-knit, depending on the number of GT.

GC is known to modulate the transcription of susceptible genes by a direct action (via the GRE) and an indirect action (via the SP1 transcription factor) $[17,18]$. It is worth noting that a perfect SP1-binding site is predicted 
on the VWF promoter when a $\mathrm{G}$ is located at position -3268 (haplotype 1), while the consensus is not ideal if a $\mathrm{C}$ is at -3268 (haplotype 2) [9]. This helps to explain why genotype $1 / 1$ represents the SNP pattern sensitive to cortisol excess, unlike genotype $2 / 2$. So we can hypothesize that the action of GC on VWF is mediated by a perfect SP1 binding and a more compact GRE. For the time being, the LD makes it impossible to determine whether both SNP haplotype and (GT) $)_{\mathrm{n}}$, or only one of them, actually modulate VWF upregulation in CS, though both were found capable of predicting the risk of developing high VWF levels.

The relationship between the effect of corticosteroids and VWF promoter polymorphisms has major clinical implications in the definition of a prothrombotic state in
CS, which is a chronic disorder associated with hypertension, hyperglycemia, dyslipidemia and obesity (now known to be severe thrombotic risk factors). The chance to predict the onset of an additional thromboembolic risk factor, such as rising VWF levels, might help us to better define hypercoagulable states and improve our management of CS patients, as well as furthering our understanding of the effect of long-term GC therapy in potentiating the hemostatic system.

Further investigations are needed, on a larger cohort of CS patients, to highlight any clinical significance of the increased SNP recombination rate in the VWF gene promoter. Given the rarity of CS, it would be useful to enlarge the cohort of CS patients by planning a multicenter study aimed to confirm our observation.

\section{References}

$>1$ Boscaro M, Barzon L, Fallo F, Sonino N: Cushing's syndrome. Lancet 2001;357:783791.

-2 Etxabe J, Vazquez JA: Morbidity and mortality in Cushing's disease: an epidemiological approach. Clin Endocrinol 1994;40:479484.

3 Van Zaane B, Nur E, Squizzato A, Dekkers OM, Twickler MT, Fliers E, Gerdes VE, Büller HR, Brandjes DP: Hypercoagulable state in Cushing's syndrome: a systematic review. J Clin Endocrinol Metab 2009;94:2743-2750.

-4 Dal Bo Zanon R, Fornasiero L, Boscaro M, Ruffato G, Luzzato G, Fabris F, Girolami A: Clotting changes in Cushing's syndrome: elevated factor VIII activity. Folia Haematol 1983;110:268-277.

5 Patrassi GM, Dal Bo Zanon R, Boscaro M, Martinelli S, Girolami A: Further studies on the hypercoagulable state of patients with Cushing's syndrome. Thromb Haemost 1985;54:518-520.

$\checkmark 6$ Fatti LM, Bottasso B, Invitti C, Coppola R, Cavagnini F, Mannucci PM: Markers of activation of coagulation and fibrinolysis in patients with Cushing's syndrome. J Endocrinol Invest 2000;23:145-150.

$>7$ Ruggeri ZM: Von Willebrand factor. Curr Opin Hematol 2003;10:142-149.

$>8$ Gill JC, Endres-Brooks J, Bauer PJ, Marks WJ, Montgomery RR: The effect of ABO blood group in the diagnosis of von Willebrand disease. Blood 1987;69:1691-1695.
9 Casonato A, Daidone V, Sartorello F, Albiger N, Romualdi C, Mantero F, Pagnan A, Scaroni C: Polymorphisms in von Willebrand factor gene promoter influence the glucocorticoid-induced increase in von Willebrand factor: the lesson learned from Cushing syndrome. Br J Haematol 2008;140:230-235.

10 Keightley AM, Lam YM, Brady JN, Cameron CL, Lillicrap D: Variation at the von Willebrand factor (VWF) gene locus is associated with plasma VWF levels: identification of three novel single nucleotide polymorphisms in the VWF gene promoter. Blood 1999;93:4277-4283.

-11 Harvey PJ, Keightley AM, Lam YM, Cameron C, Lillicrap D: A single nucleotide polymorphism at nucleotide -1793 in the von Willebrand factor (VWF) regulatory region is associated with plasma VWF:Ag levels. $\mathrm{Br}$ J Haematol 2000;109:349-353.

12 Daidone V, Cattini MG, Pontara E, Sartorello F, Gallinaro L, Marotti A, Scaroni C, Pagnan A, Casonato A: Microsatellite $(\mathrm{GT})_{\mathrm{n}}$ repeats and SNPs in the von Willebrand factor gene promoter do not influence circulating von Willebrand factor levels under normal conditions. Thromb Haemost 2009;101:298304.
-13 Hough C, Cameron CL, Notley CR, Brown C, O’Brien L, Keightley AM, Berber E, Lillicrap D: Influence of a GT repeat element on shear stress responsiveness of the VWF gene promoter. J Thromb Haemost 2008;6:11831190.

14 Arnaldi G, Angeli A, Atkinson AB, Bertagna X, Cavagnini F, Chrousos GP, Fava GA, Findling JW, Gaillard RC, Grossman AB, Kola B, Lacroix A, Mancini T, Mantero F, NewellPrice J, Nieman LK, Sonino N, Vance ML, Giustina A, Boscaro M: Diagnosis and complications of Cushing's syndrome: a consensus statement. J Clin Endocrinol Metab 2003; 88:5593-5602.

15 Casonato A, Pontara E, Sartorello F, Cattini MG, Sartori MT, Padrini R, Girolami A: Reduced von Willebrand factor survival in type Vicenza von Willebrand disease. Blood 2002;99:180-184.

16 Casonato A, Pontara E, Boscaro M, Sonino N, Sartorello F, Ferasin S, Girolami A: Abnormalities of von Willebrand factor are also part of the prothrombotic state of Cushing's syndrome. Blood Coagul Fibrinolysis 1999; 10:145-151.

17 Ou XM, Chen K, Shih JC: Glucocorticoid and androgen activation of monoamine oxidase $\mathrm{A}$ is regulated differently by $\mathrm{R} 1$ and $\mathrm{Sp} 1$. J Biol Chem 2006;281:21512-21525.

18 Hayashi R, Wada H, Ito K, Adcock IM: Effects of glucocorticoids on gene transcription. Eur J Pharmacol 2004;500:51-62. 